\title{
Automation of Big Data Analytics Using Robotic Process Automation
}

\author{
G. Malini \\ Department of Computer Science, Rabiammal Ahamed Maideen College for Women, Thiruvarur, Tamil Nadu, \\ India
}

Article Info

Volume 7, Issue 2

Page Number: 602-605

Publication Issue :

March-April-2021

\section{Article History}

Accepted : 25 April 2021

Published : 30 April 2021

\section{ABSTRACT}

Robotic Process Automation (RPA) is now becomes a buzzword and makes it mark on almost all fields in assisting automation of repetitive human intensive tasks in a simpler manner. RPA is nothing but a software solution that mimics the human interaction with computing software and applications without manual intervention. RPA has already been adapted in almost every business processes which are repetitive. As we are in the age of information the need for retrieval of patterns from raw data is increasing unimaginably so the needs for effective tools are also in a greater need. The effectiveness of RPA can be incorporated into the ever growing data analytics to automate the process of finding patterns and predictions from big data.

Keywords : RPA, Automation, Data analysis, Robotic Process Automation, Robots, Big data

\section{INTRODUCTION}

Robotic Process Automation is already have been adapted by many organizations to automate their tasks which have repeatedly done. For example in retailing they are used to categorize products, in banking sectors its used to manage customer appointments, credit cancellation, account closure and so on. In many organizations it is used to check email, make responses to those mails, and manage organizations schedules, data entry. The list of process which has been automated by RPA is endless. Among them its part in handling is more effective, because the data which has been entered or collected by using a RPA tool is most trustable, clean and structured. This capability of RPA can be further extended to do more in managing big data.

The emergence of big data largely changed the digital world the way the organizations operates. The power of big data is in finding the useful patterns which are important key in predictive analysis. Most of the business predictions are done manually which took humongous amount of time and work force which slow down the business progress. To improve the speed of data analysis automation can be incorporated into the analytic process. The technology of Robotic Process Automation is best suited for automation of processes. Since Robotic Process Automation is the ability of a machine to "do" and instruct the machine to do what humans do. 
Robotic Process Automation can be implemented from the beginning of data collection to end of pattern extraction. Data collected by Robotic Process Automation are error free and precise because when data collected manually may have human errors which have been reduced by automated bots. Since the collected data are precise then analysis on them is easier.

\section{ROBOTIC PROCESS AUTOMATION}

Robotic Process Automation is a software technology that automates the repetitive digital tasks done manually RPA enable creating bots by observing human digital actions and let them do the work. RPA bots can interact with any application or system the same way humans interact with them [11].

The term RPA is build with three important concepts,

i. Robotic - A machine mimics human actions.

ii. Process - Sequence of steps to perform a particular task.

iii. Automation - Ability of a machine to do a task without human intervention.

RPA bots act like human in understanding what's on the screen, identifying and extracting data, and so on. They mostly stick with the front end of a system.

\section{A. Misconceptions on RPA and Artificial Intelligence} (AI) [12]

There are some misconceptions about RPA that it is AI. It's not completely true because RPA is a Software bots or tool that has some extent of intelligence in them.

They are not humanoid robot or having any physical form or any resemblance to human. They cannot replicate human cognitive functions and cannot do critical or logical thinking. They do only what they are trained for.

By giving the bots AI skills they can expand their ability to handle cognitive functions like understanding structured and semi-structured data, recognize speech and make conversation and chat.

\section{B. Advantages of RPA}

i. Highly Productive - RPA bots are more productive than human because they are capable of doing work 24/7.

ii. Precise - The task done by RPA bots are precise and error free compared to manually done tasks.

iii. Cost effective - As the RPA bots works 24/7 without any vacation, the investments made on them can be returned within a short span of time.

iv. Speed - As they are piece of software and can do any task faster.

v. Improves Analytics - The data collected by RPA bots are precise and structured, which makes the process of data analysis much easier.

vi. Improved IT support and Management - RPA bots improve the functionality of service desk and other management activities.

vii. Truthfulness - RPA bots are truthful, once a task is allotted to a bot, it completes it effectively.

\section{BIG DATA ANALYTICS}

\section{A. Overview of Big data}

The technologies, tools and applications which have been used for various tasks are capable of producing enormous amount of data in both structured and unstructured forms [12]. These data are stored in large warehouses and have potential to grow exponentially by time. Day by day the size of the data increases. The data stored in them are termed as big data.

These data are not always meaningful, correct and useful; there is a need for some tools or technologies to make the data meaningful and useful. Traditional data processing tools are not capable of handling such a large amount of data.

\section{B. Analysis of big data}


There are varieties of sources that produce enormous amount of data most of them are not always useful until they are presented in a meaning full way. The data becomes meaningful when useful patterns are extracted from them. These patterns are useful in predictions and decision making and plays a vital role in business enhancement

\section{Application areas of big data analytics}

Data analytics plays a vital role in decision making [4]. Since almost every organization uses big data analytics to improve their work process.

Areas of application of big data analytics is vast, here are some areas where data analytics plays a vital role and make them understand the nature of their data and users:

i. E-Commerce

E-commerce websites analyses the customer behavior of buying and predicts some patterns form their buying history. These patterns are helpful in target promotions.

ii. Banking and financial services

Role of data analytics is of greater important in banking and financial sector. Among them are financial market monitoring, fraud detection and so on.

iii. Entertainment and media

In this industry the user preferences on audio and video are collected and analyzed for making recommendations to the users.

iv. Weather forecasting

Predicting the weather related things is very important to save many lives at the time of natural disasters. Technological advancements in forecasting with the help of big data analytics save many lives in the recent years.

v. Health care

The most important sector which widely uses data analytics is health care and medicine. Here analytics is used in extracting patterns from the history of patients to diagnose and treat rare diseases in the future.

\section{vi. Education}

Education plays a major role in producing enormous amount of data and also there is a need for analyzing and reporting using those data. These reports can be used to evaluate both teachers and students performances.

vii. Manufacturing

Big data analytics plays a major role in manufacturing industries. It's used to solve manufacturing challenges and win over competitors.

\section{Issues in Big data analytics}

However there are so many advantages in using big data analytics there are some of issues in this. The issues may include,

i. Large amount of data - data analysis helpful in so many ways when the data size increases it become difficult to do analysis.

ii. Inability of traditional data analysis tools - As the data size increases the traditional data analysis tools may fail to do its job effectively.

iii. Time consuming - As the data processing tools need manual intervention it consumes a lot of time to process huge amount of data.

iv. Poor input data - Sometimes the quality of data is poor and ambiguous which results in poor output.

v. Lots of manual interpretations needed - The tools may produce results but manual interpretation of those results in needed.

\section{ROLE OF RPA IN BIG DATA ANALYTICS}

As there were lots of issues regarding big data analytics by using traditional data processing methods and tools. To overcome these issues automation and analytical process should be incorporated.

This automation can be done with the help of RPA. As there are a lot of advantages of RPA, that can be helpful in automation of data analytical process. 
RPA has the capability in assisting big data analytics from the initial stage that is from data collection to the end stage of analysis.

RPA can be used to play variety of roles in big data analytics some of them are listed here.

i. Data input - most of the issues in data analysis can be avoided when the data entered into warehouses are entered correctly. This can be achieved by implementing RPA bots at the time of data entry.

ii. Preparing data for analysis - RPA bots are more efficient in loading and cleaning data and prepare it for analytical process [7].

iii. Analyzing and presenting the result - usually analyzing big data takes much amount of time when they are manually done. But RPA bots are much faster and scan through data quickly and efficiently thus produce results in a decent amount of time. Also it interprets the results in an understandable manner.

\section{CONCLUSION}

In recent years the growth and accumulation of data is high. However these data are not meaningful. To make those data meaningful and accessible they have to be analyzed and different patterns to be extracted. Here in this paper we have studied how data are analyzed, the areas in which data analysis play vital role, the issues in traditional data analysis process and finally the role played by RPA to overcome the issues in data analytics.

\section{REFERENCES}

[1]. D. P. Acharjya, P. Kauser Ahmed P “A Survey on Big Data Analyics: Challenges, Open Research Issues and Tools", International Journal of Advanced Computer Science and Applications (IJACSA), Vol. 7, No. 2,2016

[2]. K. P. Naveen Reddy, Undavalli Harichandrana,T. Alekhya, Rajesh S M, "A Study of Robotic Process Automation Among Artificial Intelligence",
International Journal of Scientific and Research Publications, Volume 9, Issue 2, February 2019.

[3]. Paul Roy, Peter Dickinsion, "How Robotic Process Automation and Artificial Intelligence will change Outsourcing" Seminar on June 7,2016.

[4]. Tanya Oberoi, Big Data Analytics Skills to Boost Your Salary, Great Learning Blog, Jun 1, 2018

[5]. Trung Nguyen, Intelligent Automation-A Symbiotic relationship RPA and Data Science, towards data science, April 6, 2020.

[6]. Samantha Wolhuter, Data and RPA - How Robotics is Changing Data Analytics.

[7]. Arun Goyal, Big Data Analytics: Role of Automation, Dataversity.net, February 15, 2017.

[8]. Jelani Harper, The next Level of Robotic Process Automation: Automating Data Science, Inside Big Data, April 17, 2020

[9]. Mr. Wasique Ali Ansari, Mr. Paritosh Diya, et.al, “A Review on Robotic Process Automation - The future of Business Organizations", 2nd International Conference on Advances in Science \& Technology (ICAST-2019).

[10]. Kevin C. Moffitt, Andrea M. Rozario, Miklos A. Vasarhelyi, "Robotic Process Automation for Auditing", Journal of Emerging Technologies in Accounting (2018), Volume 15, Issue 1, July 01 2018.

[11]. https://www.automationanywhere.com/rpa/roboticprocess-automation, "What is Robotic Process Automation (RPA)?", Robotic Process Automation, 30 April 2021.

[12]. Lydia Ray, "Cyber - Physical Systems: An Overview of Design Process, Applications, and Security", in Handbook of Research on Applied Cybernetics and Systems Science.

\section{Cite this article as :}

G. Malini, "Automation of Big Data Analytics Using Robotic Process Automation", International Journal of Scientific Research in Computer Science, Engineering and Information Technology (IJSRCSEIT), ISSN : 2456-3307, Volume 7 Issue 2, pp. 602-605, March-April 2021. Available at

doi : https://doi.org/10.32628/CSEIT2172124

Journal URL : https://ijsrcseit.com/CSEIT2172124 